\title{
Gastrocnaemius-propeller extended miocutanous flap: A new chimaeric flap for soft tissue reconstruction of the knee
}

\author{
M. Innocenti, E. Cardin-Langlois, G. Menichini, C. Baldrighi*
}

Reconstructive Microsurgery, Careggi University Hospital, Florence, Italy

Received 16 July 2013; accepted 7 October 2013

KEYWORDS
Lower limb
reconstruction;
Propeller flap;
Soft tissue loss around
the knee

Soft tissue defects in the knee area may result from trauma, oncologic resection and from complications due to knee replacement surgery. The knee is often involved in high-energy trauma subsequent to motor vehicle accidents,

\begin{abstract}
Summary Purpose: Soft tissue defects involving the anterior aspect of the knee are a frequent finding in a number of pathological conditions. The aim of this article is to describe a new pedicled flap consisting of a conventional medial gastrocnaemius muscle flap associated with a propeller flap based on a perforator of the medial sural artery.

Material and methods: Five males ranging in age between 26 and 72 years underwent a reconstruction of the soft tissue of the knee by means of the described procedure. Three patients sustained complex tissue loss subsequent to high-energy trauma; two losses were due to septic complications after elective knee surgery.

Results: Four flaps survived allowing adequate proximal tibial metaphysis and patella coverage. One patient underwent early above-the-knee amputation due to life-threatening septicaemia.

Discussion: The described chimaera flap consists of a medial gastrocnaemius flap with a skin paddle that is elevated on a perforator of the medial sural artery and then rotated according to the propeller flaps' principles. It provides effective coverage of large soft tissue defects of the knee. In the authors' experience, the propeller flap portion proved to be particularly useful to cover the patella, while the muscle flap was used to cover the proximal metaphysis of the tibia and fill the dead space if present.

(C) 2013 British Association of Plastic, Reconstructive and Aesthetic Surgeons. Published by Elsevier Ltd. All rights reserved.
\end{abstract}

\footnotetext{
* Corresponding author.

E-mail address: carlabaldrighi@gmail.com (C. Baldrighi).
}

and severe soft tissue injuries are frequently associated with fractures, increasing the rate of complications, particularly if internal fixation devices are used to stabilise the bone. ${ }^{1-3}$ Soft tissue sarcomas requiring a wide margin 
of resection are also frequently located in the knee area, and simultaneous reconstruction is mandatory. ${ }^{4,5}$ In the orthopaedic literature, skin infections post-total knee replacement are observed in $2 \%$ of the procedures. ${ }^{6}$ It has been proven that $58 \%$ of patients develop a deep wound infection after having a superficial surgical-site infection post-total knee or hip arthroplasty. ${ }^{7}$ In a recent study, a $0.33 \%$ complication rate of early wound healing requiring a return to the operating room was reported following total knee replacement. Although this rate is quite low, the longterm effect of these complications is important. A $6 \%$ rate of deep prosthetic infection and a 5.3\% rate of major additional surgery requirement were reported, which was much higher than for the control group (1.4\% and $0.9 \%){ }^{8}$

Particularly in case of infection, the medial gastrocnaemius muscle flap is still the best option to cover the proximal tibia. However, its arc of rotation is limited and a muscle is not the ideal tissue to cover the patella, which requires thin and pliable skin coverage.

The saphenous flap and propeller flaps based on the perforators of the deep femoral artery may be good alternatives to cover the patella region. They provide supple and pliable skin but when the defect involves the proximal tibial metaphysis, these flaps are not able to reach the recipient site to fill the dead space.

The authors report a new chimaeric pedicled flap consisting of a musculocutaneous medial gastrocnaemius flap where the skin paddle is raised, after the transfer of the muscle, according to the principles of propeller flaps, and is oriented in a different direction in order to increase the potential area of coverage and reach the patella and distal femur. This flap fulfils the requirement to cover the tibial tuberosity with a muscle flap and the patella with a skin flap.

\section{Material and methods}

From 2010 to 2012, five males ranging in age between 26 and 72 years were treated for soft tissue defects around the knee. Among these defects, three were due to trauma, one due to infection and one post-knee replacement surgery. In all these cases, due to poor general or local condition of the patient, the goal was to cover a large soft tissue defect by means of a local flap. A musculocutaneous medial gastrocnaemius flap was raised. After carrying out the first part of the dissection, the fasciocutaneous component over the muscle was then harvested to obtain a propeller flap based on the major musculocutaneous perforator from the medial sural artery. Having done that, it was then possible to rotate and place the skin paddle in a different direction from its muscle component (Figure 1).

Four patients healed uneventfully. One patient underwent emergency above-the-knee amputation, 4 days after surgery, due to life-threatening septicaemia. At the time of amputation, the flap was still viable.

\section{Surgical technique}

In case of trauma, it is recommended to perform an angiography of the lower extremity to assess the patency of the sural arteries. Preoperatively, an ultrasound Doppler is used to map the location of the musculocutaneous perforator

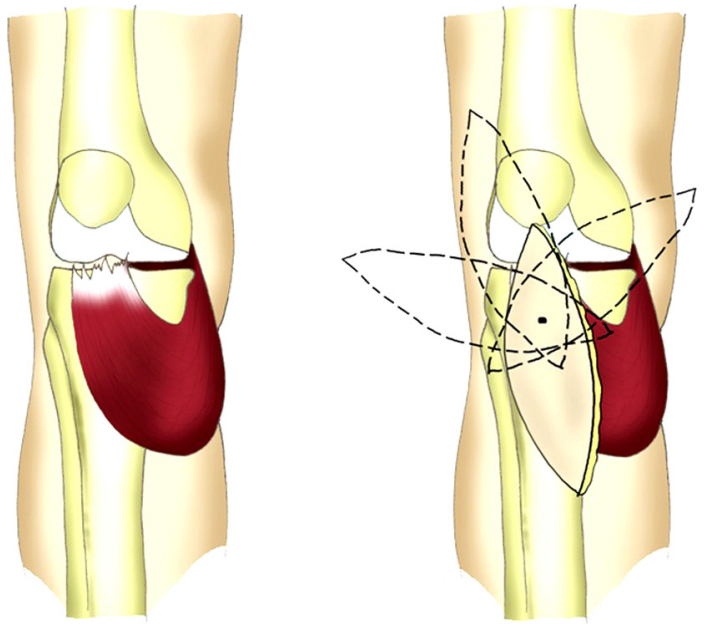

Figure 1 a) Conventional medial gastrocnemius is ideal for coverage of tibial tuberosity but it is not adequate in case of larger defects involving the patella. b) A propeller fasciocutaneous flap may be designed based on a single perforator raising from the medial sural artery and rotated in any direction. Patella is the ideal recipient site but the flap can reach also the tibial diaphysis if needed.

from the medial sural artery. One skin perforator from the medial sural artery can supply almost the whole skin above the medial gastrocnaemius muscle. ${ }^{18}$ The skin perforator is ideally located in the centre of the skin paddle but, according to the principles of propeller flaps, ${ }^{9}$ it is more conveniently located in the proximity of either the distal or the proximal pole in order to increase the arc of rotation (Figure 2). A preliminary explorative incision is made on the cutaneous projection of the medial border of the medial gastrocnaemius. The skin is elevated in a subfascial plane until the perforators are identified (Figure 3). All perforators identified during the dissection are spared until the dominant one is precisely located. Once the main perforator is identified and chosen, the flap design and skin incision are completed. Adequate vascularisation of the skin paddle is then assessed. Intramuscular dissection of the perforator is performed for $1 \mathrm{~cm}$ in order to obtain the desired arc of rotation of the skin paddle without any pedicle kinking. The skin paddle is secured to the underlying muscle to avoid shearing before raising the conventional medial gastrocnaemius muscle flap. When the muscle flap is completely raised and the muscle belly securely sutured into the recipient site, the sutures of the skin paddle can then be removed and the skin oriented in the desired direction (Figure 4 ). A $90^{\circ}$ rotation of the skin flap is often sufficient as the muscle flap covers the tibial tuberosity on a transverse axis while the skin paddle covers the residual defect on a longitudinal axis. With this technique, the authors were easily able to cover defects extending to the patella and distal femur. Primary closure at the donor site is possible for a skin defect $<6 \mathrm{~cm}$; a skin graft can be used otherwise.

\section{Case presentation}

A 32-year-old male, involved in a motorcycle accident, sustained a high-energy trauma to the left lower extremity. 


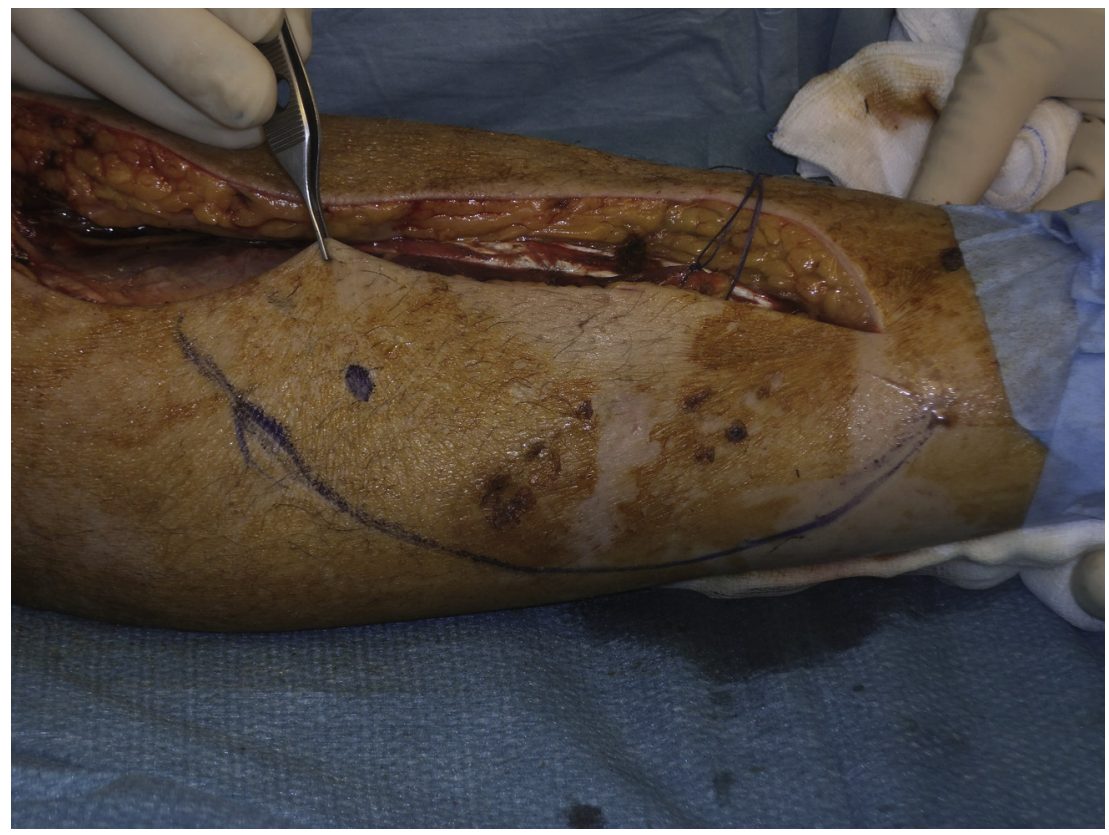

Figure 2 Preoperative Doppler investigation identifies the best perforator that is marked on the skin. The flap design includes the perforator in the proximity of the proximal pole.

He reported a severe compound open fracture involving the distal femur and proximal tibia. The Mangled Extremity Severity Score (MESS) score was $7 .^{10}$ He was initially referred to another institution where he was treated in the Emergency Department with bone stabilisation by means of a compression plate. No attempt at soft tissue coverage was done at the initial referring hospital. When referred to our Unit, 2 months after the injury, the patient presented a wide infected exposure of the knee joint, exposure of the hardware and bone necrosis (Figure 5). Radical resection of the knee joint, temporary replacement with a cement spacer (Figure 6) and soft tissue coverage were planned. A chimaeric flap composed of a medial gastrocnaemius flap augmented with a propeller flap, based on a perforator of the medial sural artery, was used to reconstruct the soft tissue. The muscular belly was rotated to cover the proximal tibia and a skin flap, $18 \mathrm{~cm}$ long and $12 \mathrm{~cm}$ wide, was then elevated and rotated $90^{\circ}$ to cover the knee joint and the distal femur (Figure 7). Both flaps survived and healed in 15 days achieving a reliable coverage of the cement spacer and the exposed bone (Figure 8). A skin graft was needed to close the donor site (Figure 9).

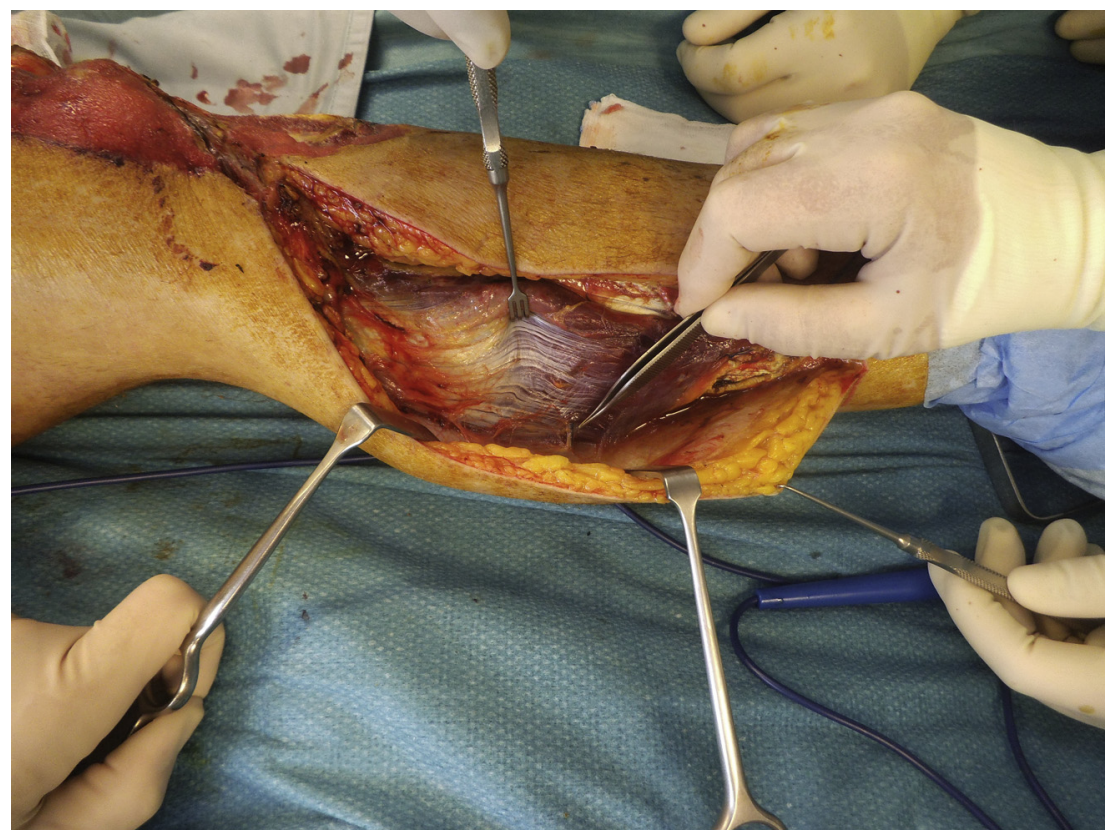

Figure 3 A straightforward dissection in the sub-fascial plane allows for direct visualization of the best perforator. 


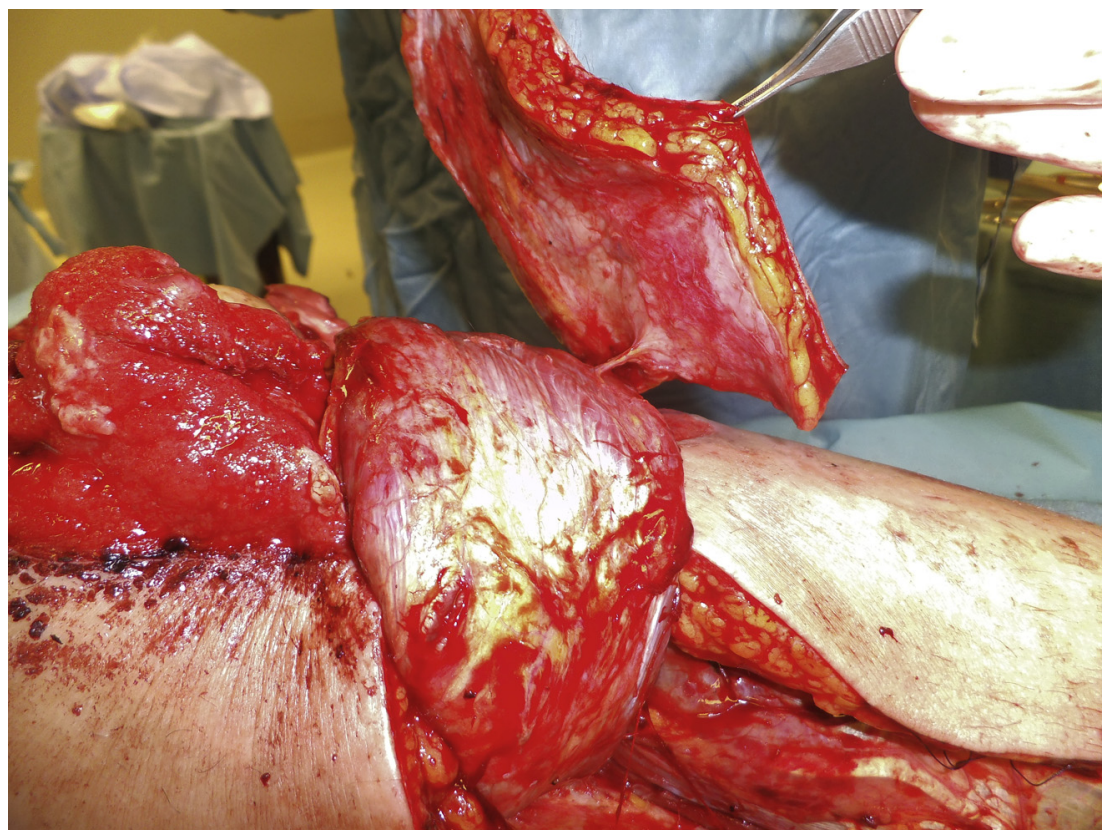

Figure 4 The muscle is secured to the tibial metaphysis and the skin paddle may be rotated on a single perforator according to the propeller flaps principles.

At 3 months' follow-up, both the donor and recipient sites are healed (Figure 10).

\section{Discussion}

Adequate coverage of the knee is often a challenge for the reconstructive surgeon. Local options are quite limited in terms of size and are often restrained by their arc of rotation. However, free flaps may increase the length and complexity of surgery. Whenever possible, salvage of the knee joint is highly advocated, as above-the-knee amputation is associated with a high complication rate and increase in energy expenditure for the patient. It is well established that muscle flaps are best indicated to fill the dead space when present; besides, the high vascular volume of these flaps serves as a carrier for antibiotics locally. Fasciocutaneous flaps, though, provide thin and pliable skin offering a good, deep gliding surface over the patella and are, therefore, preferred to cover the anterior aspect of the knee joint. The workhorse flap to cover defects following infection, trauma and tumour excision in the anterior aspect of the knee is still the medial gastrocnaemius muscle flap. It provides good vascularised tissue that helps sterilise the recipient site, it is wide and bulky enough to cover a prosthesis and fill the dead space if needed, and it can be transferred as a musculocutaneous flap to provide skin coverage. Unfortunately, the medial gastrocnaemius flap cannot easily reach the upper portion of the knee. In addition, the conventional musculocutaneous flap, raised along with the skin extension over the medial gastrocnaemius, is quite bulky and the cutaneous portion being attached to the underlying muscle cannot be freely and independently placed to increase the surface of the flap.

To increase the length of the flap, different techniques have been reported. These techniques mainly entail scoring the underlying muscle fascia and/or dissecting the muscle up to its origin. Whitney et al. reported on an angiographic study performed to identify the origin of the sural arteries in relation to the joint space. According to this article, the origins of the arteries were located above the joint line in all the cases (mean of $32 \pm 14.5 \mathrm{~mm}$ above the lower femur). The medial gastrocnaemius muscle was mobilised up to 21 and $26 \mathrm{~cm}$, respectively, above the joint line to

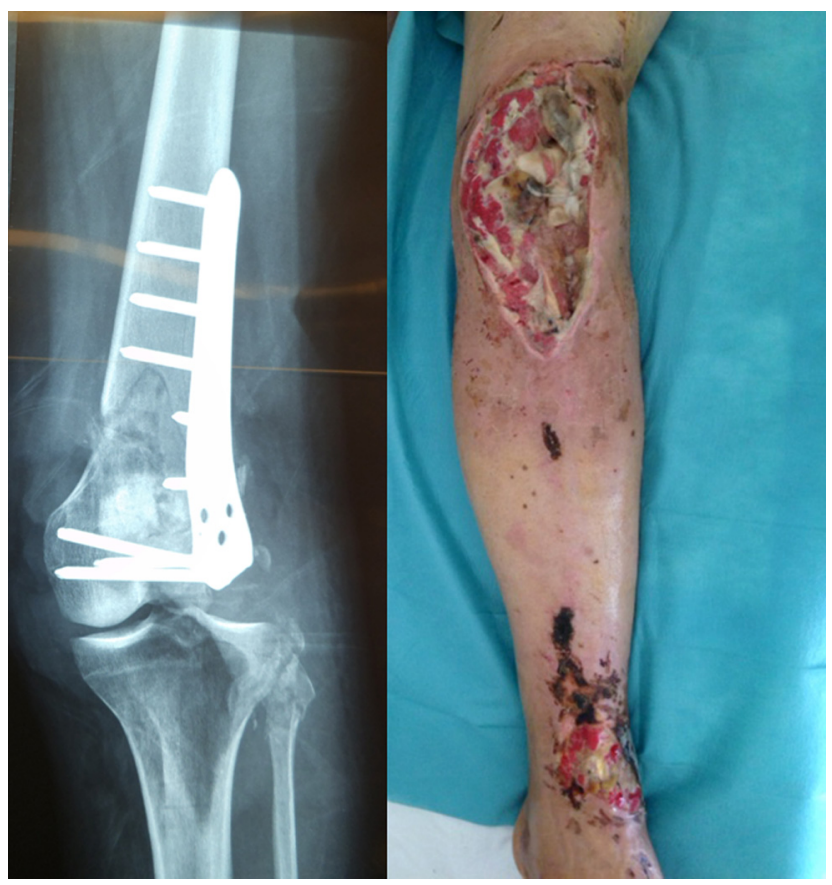

Figure 5 Clinical and Radiographic appearance at the time of referral. The lateral condyle of the femur has been avulsed by the trauma. Large soft tissue defect, infected exposure of the knee joint, exposure of the hardware and bone necrosis. 


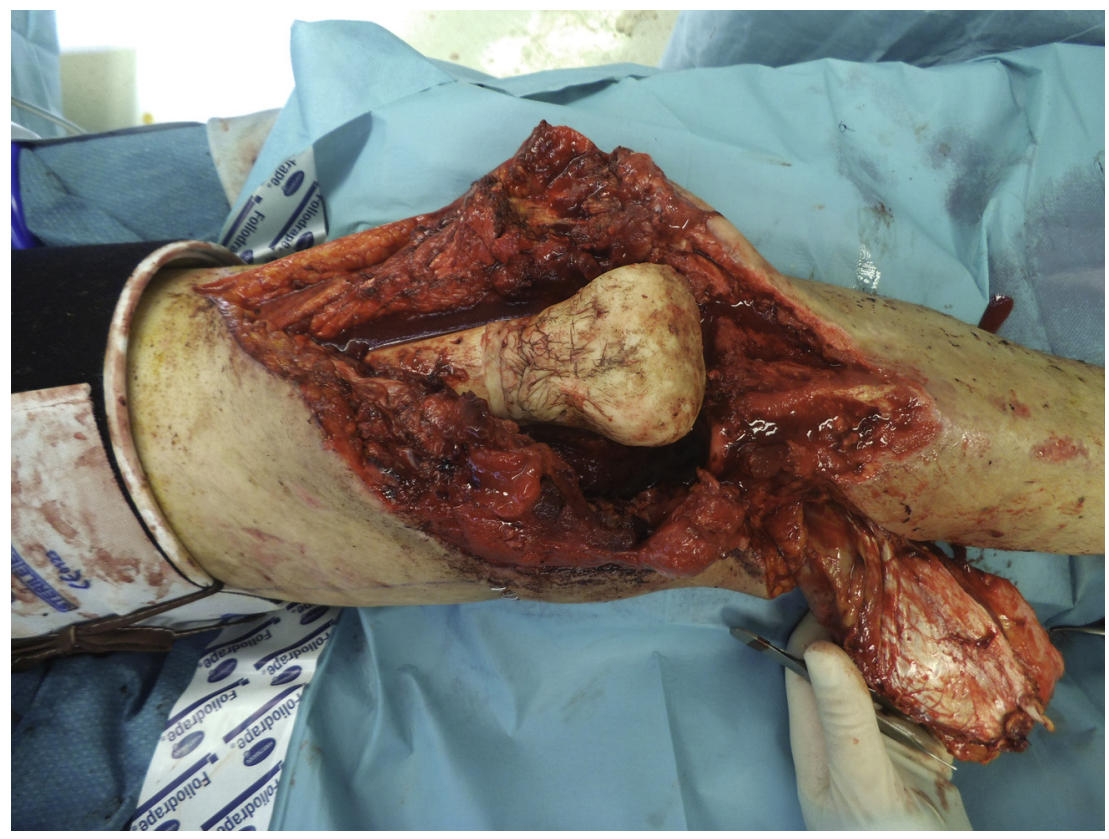

Figure 6 Radical debridement of the distal femur and the joint. Custom made cement spacer.

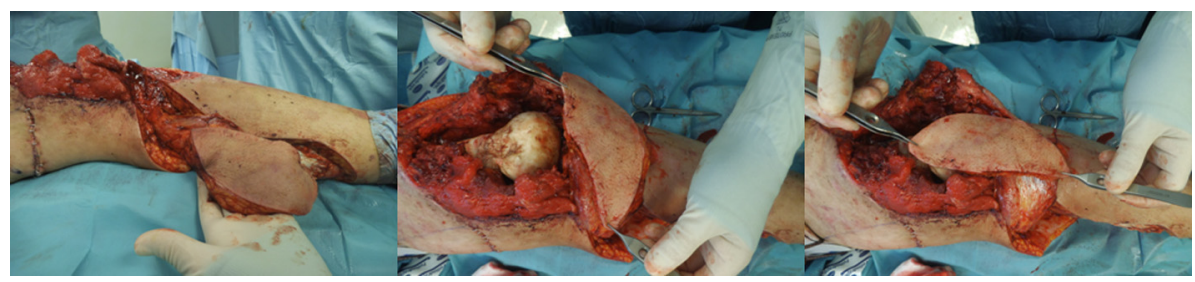

Figure 7 The mio-cutaneous flap is elevated and transferred into the recipient site. The skin paddle is then rotated upward to cover the cement spacer, which replaces the distal femur.

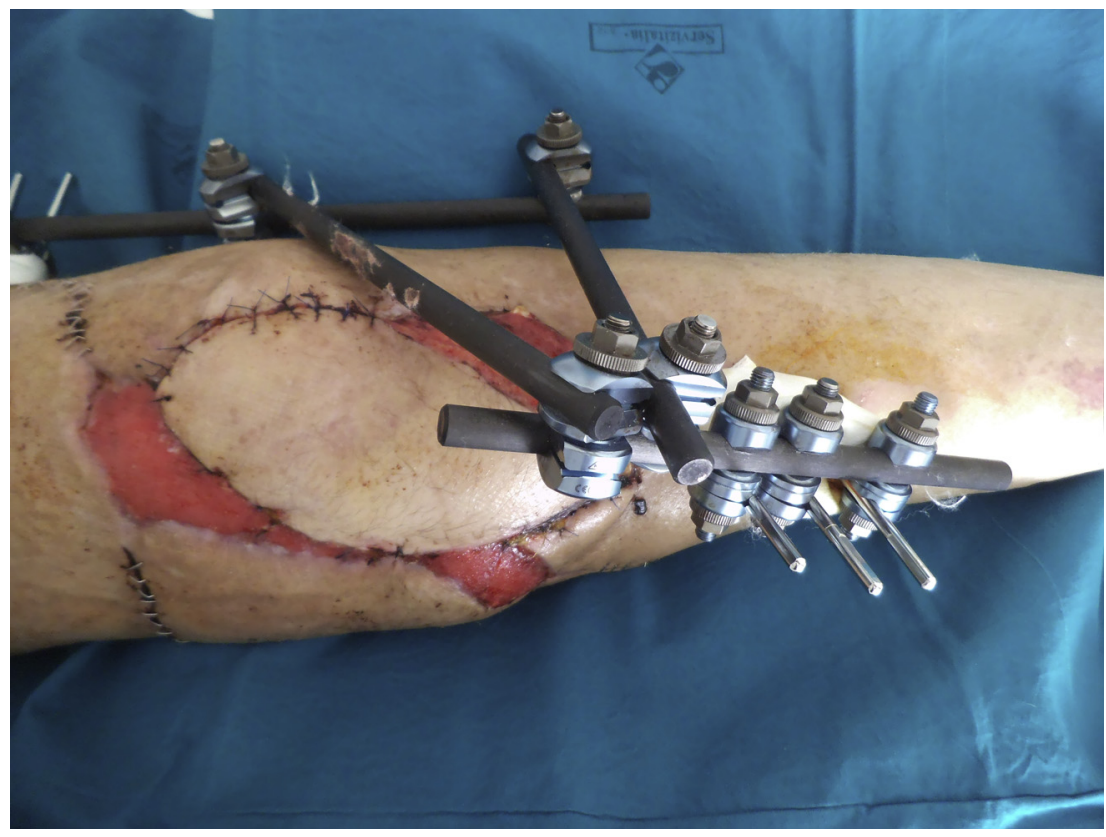

Figure 8 Clinical outcomes four weeks after surgery. 


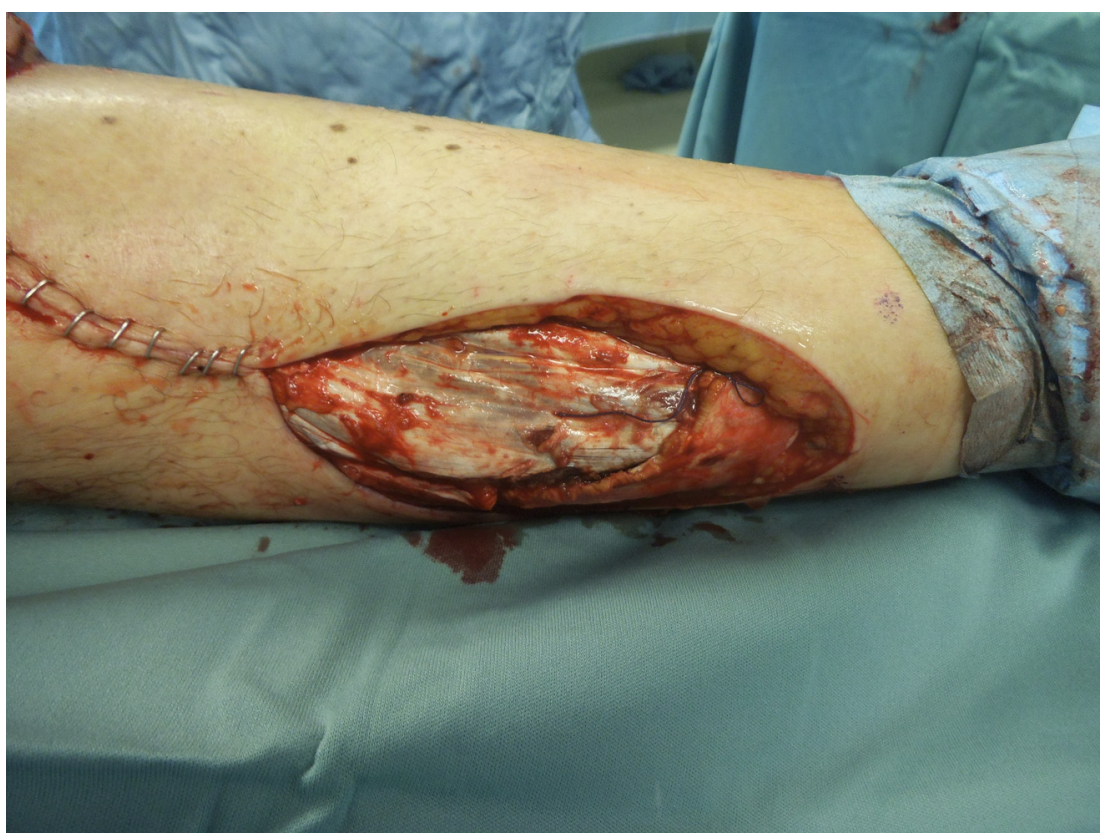

Figure 9 Donor site.

cover two defects. ${ }^{11}$ In our experience, nonetheless, proximal dissection of the pedicle may be tricky and time consuming and it is often difficult to achieve a good coverage for the upper knee area. In addition, the muscle tends to adhere to the patella instead of providing a smooth gliding surface.

Propeller flaps have been extensively used for soft tissue reconstruction in almost all the anatomical districts. ${ }^{12,13}$ They can be raised virtually anywhere a suitable perforator is available and they follow simple principles. The eccentric location of the pedicle and the possibility to rotate the flap as much as $180^{\circ}$ allow for a great coverage potential of small- to-medium-sized defects according to the principle of 'repair like with like'. The morbidity at the donor site is minimal. Any axial artery of an anatomical region (source artery) gives perforators able to supply skin flaps. In this case, the source artery is the medial sural artery and it is within the medial gastrocnaemius muscle. In 1996, Montegut ${ }^{14}$ first described the clinical application of the medial sural artery perforator flap. The anatomical background, however, dates back to the 1970s when Taylor and Daniel investigated the surgical anatomy of the region considering possible donor sites for free flaps. ${ }^{15}$ More recently, Hallock pointed out the availability of this pedicled flap to reach, as far as the medial gastrocnaemius muscle flap itself was concerned, the knee and upper third of the leg defects. ${ }^{17}$ Lately, several anatomical studies have been carried out to define the number, size and location of these perforators. ${ }^{16-19}$ According to Wong et al., in 2012, the medial sural artery gives off two branches, a medial one and a lateral one (the lateral being the one towards the midline of the muscle). Two to six perforators are identified, from 10 to $16 \mathrm{~cm}$ from the popliteal crease, with a mean of $10 \mathrm{~cm}$ from the popliteal crease for the larger one. The perforators from the lateral row are larger and located, in average, $2 \mathrm{~cm}$ from the midline. ${ }^{19}$ Perforators from the lateral row are also more frequent, being present in $66 \%$ of the dissections versus $34 \%$ from the medial row. ${ }^{18}$ The lateral sural artery system and its perforators, which supply the medial gastrocnaemius muscle and the overlaying skin, are much less reliable, being present in only $20 \%$ of the dissections. ${ }^{16}$ The skin perforator from the medial sural artery is able to supply almost the whole skin overlying the muscle, with an average area of $13 \times 8 \mathrm{~cm} .^{18}$ Although the abovementioned anatomical studies attempt to provide surgical landmarks for the identification and dissection of perforators, it is our opinion that any single patient should be preoperatively investigated by a Doppler ultrasound. The best perforator should then be identified and marked on the skin before surgery.

\section{Conclusions}

The authors present a single reconstructive solution for soft tissue loss around the knee, which combines the workhorse gastrocnaemius muscle flap with its skin perforator component, both based in the medial sural artery. The described technique allowed a substantial increase of the flap surface and therefore the coverage potential (especially in the lower femur area), avoiding the use of a second local thigh perforator flap or a free flap.

Previous articles have described the medial gastrocnaemius muscle flap and also the medial sural perforator flap, but no studies have combined the two techniques to provide a chimaeric flap. The presented flap has proven to be extremely useful for knee reconstruction where the defect is too large for a single medial gastrocnaemius muscle flap. In our preliminary experience, the best indication for this flap is in defects involving the tibial tuberosity and extending proximally up to the quadriceps tendon. In these cases, the muscle easily achieves a safe coverage of the proximal tibia, while the skin paddle, 


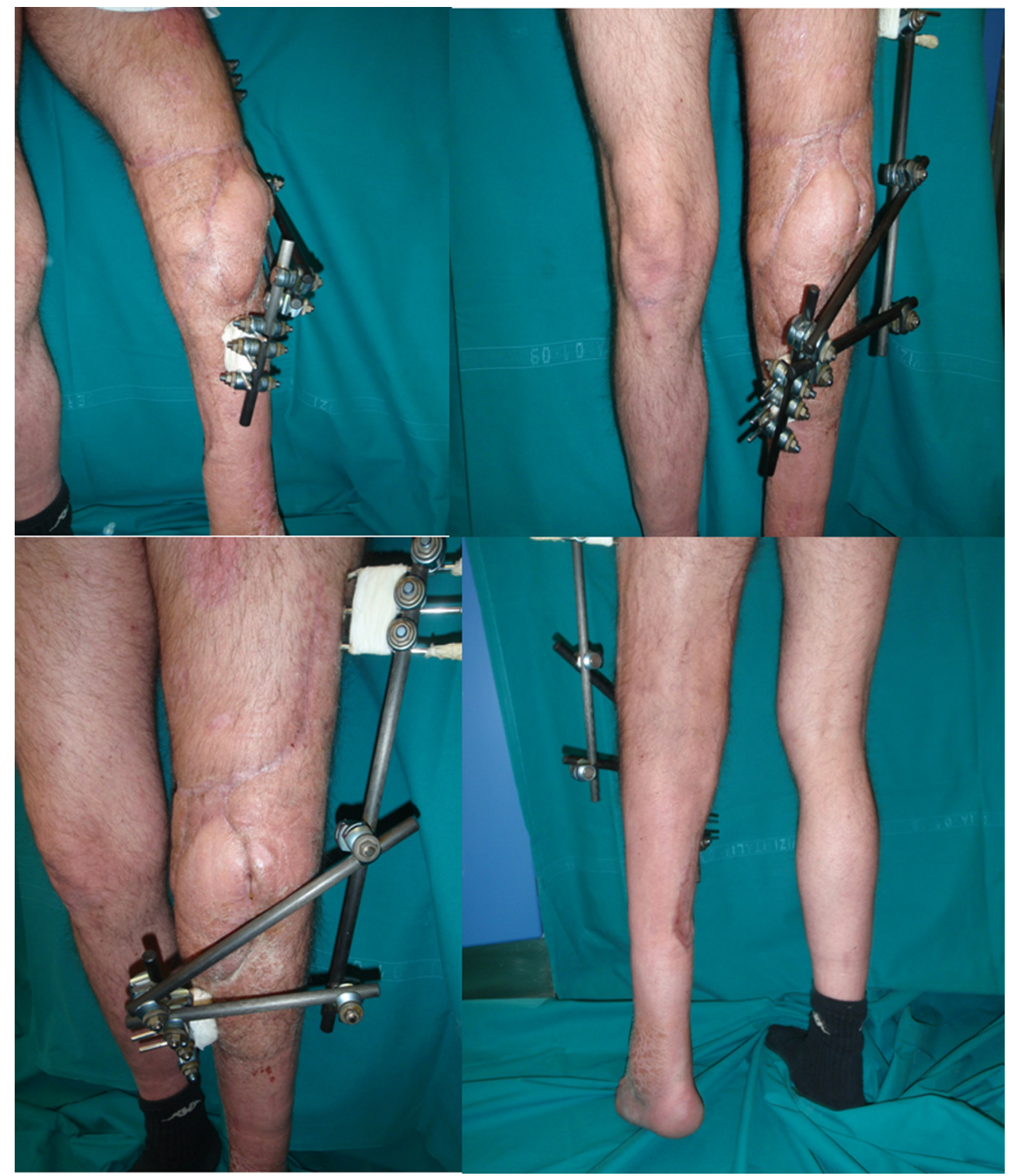

Figure 10 Clinical outcomes at 3 months follow-up.

rotated upwardly of $90^{\circ}$, provides excellent, thin and pliable skin to cover the patella. In addition, in case of defects involving the tibial diaphysis, the skin flap may be rotated distally, achieving optimal reconstruction of the soft tissue overlying the tibial crest.

In conclusion, this flap has proven to be versatile and highly reliable, offering an alternative solution to reconstruct medium- tolarge-sized defects around the knee. Its anatomy is consistent and the dissection relatively straightforward.

\section{References}

1. Covall DJ, Fowble CD, Foster TE, Whitelaw GP. Bicondylar tibial plateau fractures: principles of treatment. Contemp Orthop 1994;28(2):115-22.

2. Spagnolo R, Pace F. Management of the Schatzker VI fractures with lateral locked screw plating. Musculoskelet Surg 2012; 96(2):75-80.
3. Phisitkul P, McKinley TO, Nepola JV, Marsh JL. Complications of locking plate fixation in complex proximal tibial injuries. $J$ Orthop Trauma 2007;21(2):83-91.

4. Singer S, Eberlein TJ. Surgical management of soft tissue sarcomaln Advances in surgery, vol. 31. St. Louis: Mosby-year Book, Inc.; 1997. p. 395-420.

5. Singer S, Demetri GD, Baldini EH, Fletcher CD. Management of soft-tissue sarcomas: an overview and update. Lancet Oncol 2000 Oct;1:75-85.

6. Hanssen A, Rand J. Evaluation and treatment of infection at the site of a total hip or knee arthroplasty. AAOS 1999;48: 111-22.

7. Saleh K, Olson M, Resig S, et al. Predictors of wound infection in hip and knee joint replacement: results from a 20 year surveillance program. J Orthop Res 2002;20:505-15.

8. Galat DD, McGovern SC, Larson DR, Harrington JR, Hanssen AD, Clarke HD. Surgical treatment of early wound complications following primary total knee arthroplasty. JBJS 2009;91: $48-54$.

9. Teo TC. The propeller flap concept. Clin Plast Surg 2010;37(4): 615-26. 
10. Johansen K, Daines M, Howey T, Helfet D, Hansen Jr ST. Objective criteria accurately predict amputation following lower extremity trauma. J Trauma 1990;30(5):568-72 [discussion 572-573].

11. Whitney TM, Heckler FR, White MJ. Gastrocnemius muscle transposition to the femur: how high can you go? Ann Plast Surg 1995;34(4):415-9.

12. Gir P, Cheng A, Oni G, Mojallal A, Saint-Cyr M. Pedicledperforator (propeller) flaps in lower extremity defects: a systematic review. J Reconstr Microsurg 2012;28(9):595-601.

13. Lecours C, Saint-Cyr M, Wong C, et al. Freestyle pedicle perforator flaps: clinical results and vascular anatomy. Plast Reconstr Surg 2010;126(5):1589-603.

14. Montegut WJ, Allen RJ. Sural artery perforator flap as an alternative for the gastrocnemius myocutaneous flap. In: Proceeding from the 90th annual scientific assembly of the southern medical association, Baltimore, MD November 20-24, 1996.

15. Taylor GI, Daniel RK. The anatomy of several free flap donor sites. Plast Reconstr Surg 1975;56:243-53.

16. Cavadas PC, Sanz-Giménez-Rico JR, Gutierrez-de la Cámara A, Navarro-Monzonís A, Soler-Nomdedeu S, Martínez-Soriano F. The medial sural artery perforator free flap. Plast Reconstr Surg 2001;108(6):1609-15.

17. Hallock G. Anatomic basis of the gastrocnemius perforatorbased flap. Ann Plast Surg 2001;47(5):517-22.

18. Thione A, Valdatta L, Buoro M, Tuinder S, Mortarino C, Putz R. The medial sural artery perforators Anatomic basis for a surgical plan. Ann Plast Surg 2004;53(3):250-5.

19. Wong MZ, Wong CH, Tan BK, Chew KY, Tay SC. Surgical anatomy of the medial sural artery perforator flap. J Reconstr Microsurgery 2012;28(8):555-9. 